Review

\title{
Research Following a Foundation in the Applied Optics Group at University of Kent
}

\author{
Alan D. KERSEY \\ Technology Venture Consultant, Glastonbury, Connecticut 06073, USA \\ *Corresponding author: Alan D. KERSEY_ E-mail: Photonics1@aol.com
}

\begin{abstract}
This paper summarizes research activities at University of Kent over the period from September 1981 through November 1984. Subsequent researches undertaken in the US Naval Research Laboratory and two start-up companies are also described.
\end{abstract}

Keywords: Interferometry, polarization, multiplexing, Bragg gratings

\section{A foundation built at Kent}

I started my new post-doctoral position with Prof. David Jackson in the autumn of 1981. My first task in the newly formed Applied Optics Group was to paint the walls of the recently acquired optical labs; not quite what I was expecting, but nevertheless, being young, eager and interested in exploring new frontiers in fiber optic sensing, my new post-doctoral colleague, Mike Corke, and I pressed on. As I reflect back now, it was as if we were starting our own company - the spirit was entrepreneurial \& we were going to wear any hat required to get the job done.

Our focus at Kent started with the topic of demodulation techniques for interferometer sensors based primarily on Mach-Zehnder (MZ) configuration. Early experiments utilized bulk beam splitters to split the incident laser beam into two separate fiber paths. These paths were then used as reference and sensor arms, typically with piezoelectric transducers used to simulate weak acoustic signals in the sensing path. Prof. Jackson addressed the signal fading in interferometric sensors by demonstrating a heterodyne detection, and phase-locked homodyne approaches during his sabbatical year in Naval Research Laboratory (NRL) [1, 2]. These approaches provided wide dynamic range, but due to the fact electrically active elements were included in the arms of the interferometer, they were impractical for use in many applications of interest at the time, such as underwater acoustic sensing. This motivated a research thrust to explore interferometric demodulation techniques based on a class of "synthetic", or "pseudo" heterodyne [3] and passive homodyne approaches [4]. The advent of low cost laser diodes - driven largely by the $\mathrm{CD}$ reader industry - allowed for a variety of approaches based on laser-modulated interrogation techniques. Figure 1 shows the output of an unbalanced Mach-Zehnder interferometer driven by an 830-nm diode laser which is frequency swept via injection current modulation. The resulting sweep over a full interferometric "fringe" with a rapid flyback provided the basis for a "pseudo-heterodyne" demodulation scheme, which was one of the first practical passive interrogation approaches for interferometric sensors. These techniques were also

Received: 1 January 2011 / Revised version: 30 January 2011

(C) The Author(s) 2011.This article is published with open access at Springerlink.com 
very successfully adapted to a variety of applications over the years spent at Kent, including surface vibrometry [5] and Sagnac interferometric gyroscope configuration [6].

As mentioned previously, much of our initial work was done using bulk beam splitters - fiber based couplers were in short supply, as the technology to taper \& fuse single mode fibers to form 3-dB couplers had only just been developed. Mike Corke worked diligently at Kent to build a fused-coupler fabrication station and successfully produced devices for the research efforts. In 1983, this led to the first all-fiber Michelson interferometer (Fig. 1), being reported. The paper, which described the use of the interferometer, combined with a digital fringe counter, as a temperature sensor was first reported as a post-deadline paper at the 1st International Conference on Optical Fiber Sensors (OFS-1) held in London in 1983 [7].

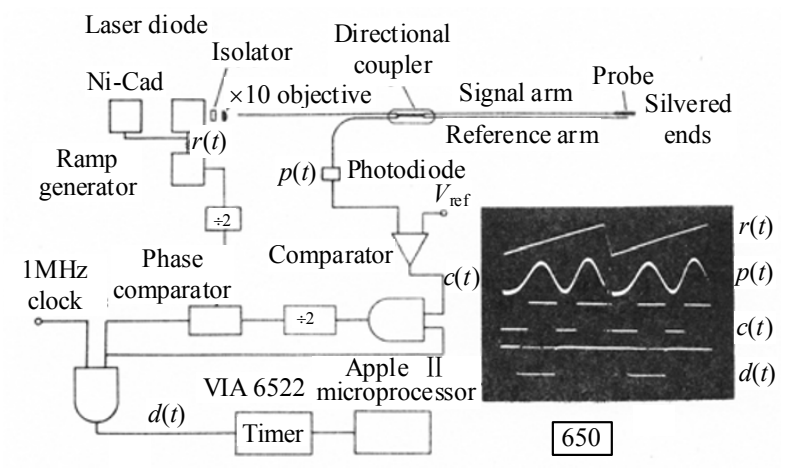

Fig. 1 All-fiber Michelson interferometer, first reported in 1983.

In addition to the fundamental signal processing of interferometric signals for demodulation, we also made some great first step in creating interferometric accelerometer [8] and magnetometer configurations $[9,10]$. The magnetometer was particularly noteworthy as the concept relied on a closed loop approach that stabilized the bias magnetic field around a magnetostrictive element attached to the fiber in one arm of a MZ interferometer. This technique eliminated the issue of hysterisis common to magnetostrictive elements, and provided for the first time a sensitive, reproducible fiber optic based magnetic field sensor.

Finally, in collaboration with several other fellow researchers at Kent, we extended these concepts to explore fiber Fabry-Perot(FP) sensors, polarimetric sensors based on Hi-Birefringence fiber, and again tested these new configurations for sensing a variety of measurands [11-14].

\section{Interferometric sensing}

In November 1984, I took up a position in NRL in Washington DC. NRL's optical science research team had been leading the way in developing fiber optic sensors for underwater acoustic sensing, and had already demonstrated many new acoustic transduction and interferometer phase measurement techniques, much of which was reported in a seminal article by T. Giallorenzi et al. in 1982 [15].

My initial work in the NRL continued to explore the fundamentals of interferometers and develop techniques to optimize the performance of sensor systems, particularly sensor arrays. This work focused on several key areas:

1) demodulation and noise performance,

2) polarization effects in interferometers,

3) multiplexing architectures, and

4) "DC" measurands.

The following subsections describe some of the key accomplishments in these areas.

\subsection{Demodulation \& phase noise}

The importance of developing passive demodulation techniques for interferometric sensors to support acoustic sensor development work in NRL leads to a focus on practical interrogation and noise reduction techniques. With laser modulated based interrogation, an unbalanced interferometer is required, which leads to an increase in phase noise in the interferometer output, limiting the sensitivity that could be achieved. The research undertaken explored methodologies to compensate for the noise, and to test new laser sources for a device with promising characteristics. Novel demodulation \& phase noise compensation approaches were 
developed as part of this activity [16, 17]. One simple and novel approach utilized a "cosine generator" chip (Analog Devices AD639), which could be phase locked to the output of an interferometer, and thus track the interferometer phase bias through a simple electronic closed loop approach [18]. This technique was very successfully applied to the fiber gyroscope, and implemented in a flight-tested version of the device [19].

As an example of the testing of laser sources, newly developed YAG ring lasers [20] were found to be particularly suited for use in interferometric sensor systems due to their exceptionally low frequency jitter and ability to be frequency modulated at high rates. The noise characteristics of these lasers were 2 to 3 orders of magnitude lower than that of diode lasers, and proved to be valuable tools for a range of subsequent work in this field. This laser also provided the basis for an interferometric noise measurement experiment that explored the intrinsic thermal noise in the fiber itself [21]. In this experiment, a MZ interferometer, with fiber arms of $1 \mathrm{~km}$ each in length, balanced within about $1 \mathrm{~cm}$. The interferometer was built in an acoustic \& vibration isolation housing to minimize extraneous noise. The noise spectrum was recorded and calibrated against a known phase reference signal. The fiber arms were then carefully cut back to $100 \mathrm{~m}$ and $10 \mathrm{~m}$ each (approx.) while maintaining an unbalance of $1 \mathrm{~cm}$ between the paths, and the phase noise spectrum was recorded. The phase noise floor was found to be proportional to $\sqrt{ } L$, in good agreement with a theory developed. These experiments reported some of the lowest phase noise level in fiber interferometric systems $(<0.1 \mu \mathrm{rad} / \sqrt{ } \mathrm{Hz}$ at $1 \mathrm{kHz})$.

Noise effects in reflective interferometric configurations such as Michelson interferometer were analyzed and an approach to eliminate the effects of coherent Rayleigh backscatter was developed [22].

\subsection{Polarization effects}

Polarization effects are also a critical factor in interferometric sensing, as polarization drifting can lead to a phenomena termed polarization induced fading, whereby the visibility of an interferometer output can be reduced to zero for orthogonal states exiting the arms of an interferometer. While this was widely understood [23], and polarization controllers based on rotatable "fiber waveplates" were developed for use in laboratory experiments [24], the phenomenon created a serious practical limitation for remotely deployed sensor systems. As part of the "core" activity in NRL, my co-workers \& I extensively studied, characterized and modelled polarization effects in interferometric sensors [25, 26]. This work led to the development of new approaches to eliminate polarization fading based on input polarization tracking [26], scanning [27], and switching [28] techniques. A full statistical model for the fading of interference visibility in interferometers was developed which allowed an accurate assessment of the probability distributions for fading conditions [29]. This work provided valuable insights into the polarization behaviour of interferometric sensors, and new active control mechanisms for overcoming the effect.

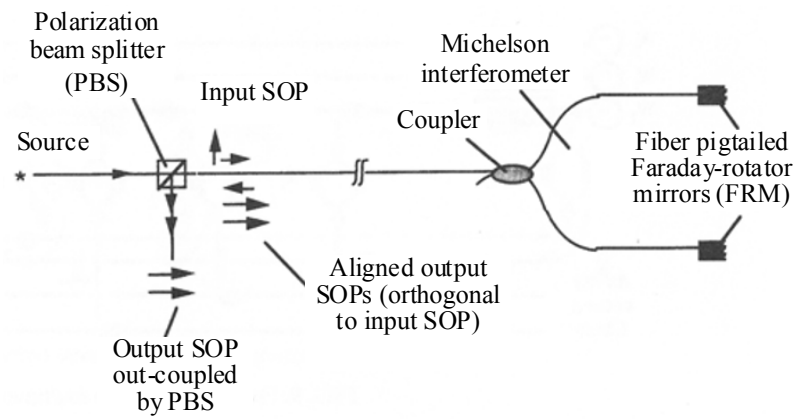

Fig. 2 Polarization-insensitive Michelson interferometer based on the use of Faraday-rotator mirrors (FRMs).

In a related activity, the application of this understanding to Michelson interferometers configured with FRMs, as apposed to conventional mirrors, led to the demonstration of a completely polarization independent interferometric system [30, 31]. This unique configuration, illustrated in Fig. 2 was subsequently widely used due to its practical 
aspects. The work was also extended to explore the use of depolarized input light in interferometry [32], gyropscopes [33], phase noise induced arising due to input polarization fluctuations $[34,35]$, and create novel formats of polarization independent ring-resonators for example [36].

\subsection{Multiplexing techniques}

Many of the applications for interferometric fiber sensors developed in NRL focused on the use of arrays of such devices, and this led to the development of a variety of multiplexing approaches. This is particularly true for interferometric acoustic sensor (hydrophone) arrays, but applied also to magnetic sensors arrays.

Multiplexing approaches based on time (Fig. 3) $[37,38]$, frequency [39], code [40], and wavelength-time division [41] techniques were developed and implemented. In many cases, these approaches are analogues of the multiplexing techniques developed for optical fiber communication systems, and consequently, leveraged many of the fiber based modulators, frequency shifts and wavelength combiners \& splitters developed for communication in order to implement these techniques.

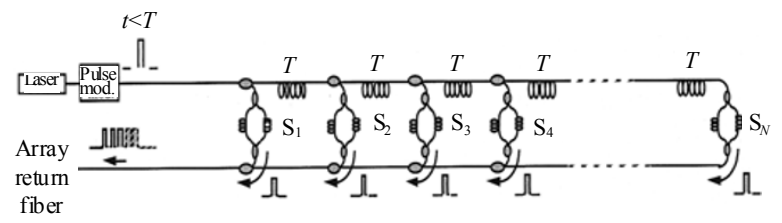

Fig. 3 Time division multiplexed array of Mach-Zehnder interferometers.

Each of these multiplexing schemes was implemented, extensively tested, and compared [42, 43]. The most capable multiplexing formats developed have exploited the power of hybrid approaches - e.g., by combining time and wavelength division multiplexing [41]. Time- and wavelength-division multiplexed arrays were ultimately developed and demonstrated for multiplexing over 120 sensors. Several novel configurations for multiplexed sensor formats that did not employ completely separated distinct interferometric sub elements were also devised \& demonstrated [44-46].

\section{4 "DC" measurands}

Another focus of the work in NRL was in understanding and developing methodologies to utilize interferometric sensors for measuring "DC" measurands, such as quasi-static strain, acceleration. These techniques utilized multi-spectral, dual-wavelength or non-linear transduction mechanisms to allow the detection of phase in an extended unambiguous dynamic range. The first demonstration of a dual wavelength interferometric approach applied to MZ interferometers [47], fiber gyroscopes [48], and polarimetric sensors [49] was reported. Approaches based on sub-carrier modulation [50], and coherence synthesis/visibility control were also devised \& demonstrated [51].

The nonlinear phase transduction approach developed [52] utilized a novel geometric arrangement for detecting strain in which a fiber sensing element was moved laterally from its longitudinal axis, as indicated in Fig. 4.
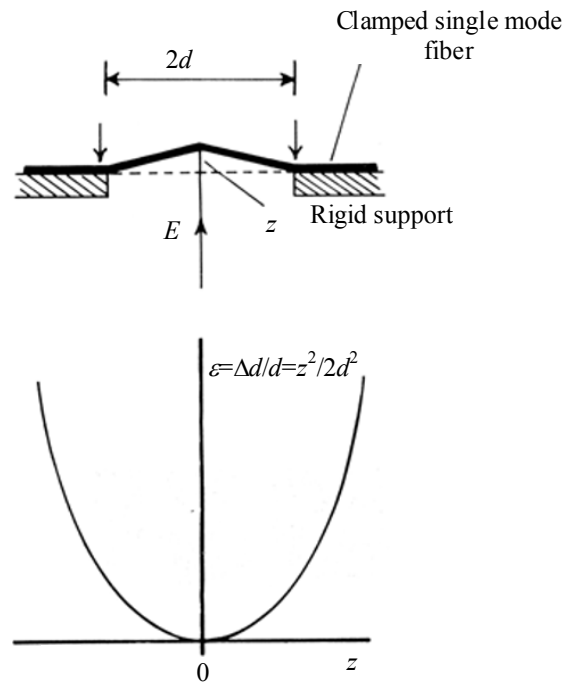

Fig. 4 Non-linear phase transduction mechanism for "DC measurand" sensors.

This created a quadratic dependence on the fiber strain on displacement, which was exploited to provide DC measurement capability through a high frequency dither applied to the fiber. The technique was used to 
create a number of different sensor configurations, including an accelerometer with sub- $\mu$ g sensitivity and excellent stability [53], and a DC pressure sensor [54]. Multiplexing concepts for these forms of DC measurands were also devised \& tested $[55,56]$.

In addition to the work on the fundamental operational characteristics of interferometric systems, the application of these interferometers to a range of practical sensor configurations was also explored. Much of this work exploited attributes of the various signal processing/demodulation concepts to create novel magnetic field [57-59] and Faraday rotation current sensors $[60,61]$.

\section{Bragg grating sensors}

Fiber Bragg gratings (FBGs) are intrinsic sensor elements that can be "written" into optical fibers via a ultra-violet (UV) photo-inscription process. The photo-inscription process produces a periodic modulation of the index of the glass in the fiber, which has been shown, with appropriate annealing, to be stable even at relatively elevated temperatures. The advantages of Bragg grating sensors are well known in the fiber sensor community, and include: providing a simple intrinsic "in-fiber" sensing element, their inherent self-referencing capability, and ease of multiplexing along a single fiber [62].

FBG based sensors have proven to be useful for a variety of applications. In particular the field of embedded distributed sensing in materials for creating "smart structures" has been a primary interest. Here fiber sensor arrays can be embedded into the materials to allow measurement of parameters such as load, strain, temperature, and vibration, from which the health of the structure can be assessed and tracked on a real-time basis. FBGs have also been explored as the optical sensing element in a range of other fiber sensor configurations; grating based chemical sensors, pressure sensors, and accelerometers are examples [62].

As illustrated in Fig. 5, the basic principle of operation commonly used in a FBG based sensor system is to monitor the shift in wavelength of the returned "Bragg" signal with the changes in the measurand (e.g. strain, temperature). The Bragg wavelength, or resonance condition of a grating varies approximately linearly with temperature (over $0-200{ }^{\circ} \mathrm{C}$ ) and strain. A general run of thumb responsivity is a change of approximately $1 \mathrm{pm}$ $(0.001 \mathrm{~nm})$ per $\mu$ strain and approximately $9 \mathrm{pm}$ per $1{ }^{\circ} \mathrm{C}$ for Ge-doped silica fibers at $1.3 \mu \mathrm{m}$.

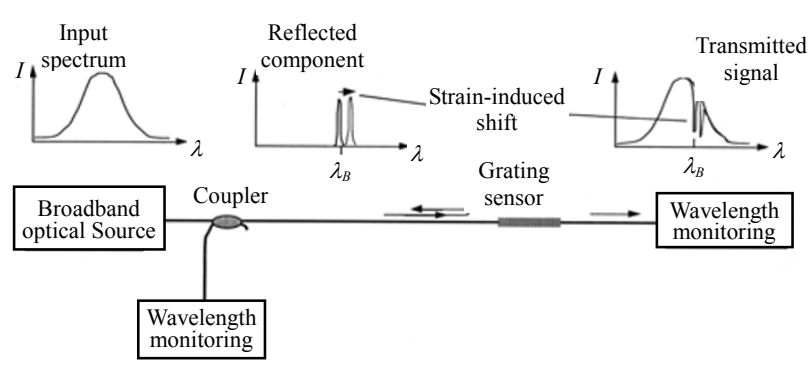

Fig. 5 Fiber Bragg grating spectral response.

The work on FBGs reviewed here falls into three categories:

1) interrogation and multiplexing approaches,

2) applications in structural monitoring, and

3) FBG sensor system commercialization.

\subsection{Interrogation and multiplexing approaches}

Although the applicability of FBGs to sensing was demonstrated by Meltz et al. in 1989 [63], the experimental system utilized laboratory spectrum analyzers for the wavelength determination. Following this, approaches based on simple wavelength discriminators were reported [64], employing ratio-metric detection and broadband filter concepts. The first practical interrogation approach for a multiplexed string of FBGs, illustrated in Fig. 6, based on the use of a scanning fiber Fabry-Perot filter was reported in 1990 [65].

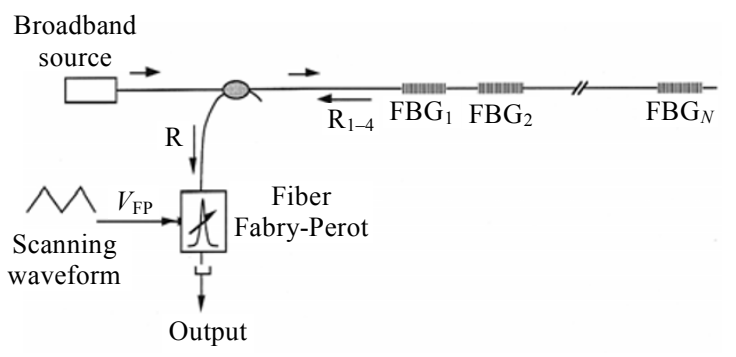

Fig. 6 Scanning Fabry-Perot based multiplexed FBG sensor array. 
Figure 7 shows a comparison of the strain monitored with a scanning-filter demodulated FBG and a resistive foil strain gauge reference gauge when both were strained to $-2000 \mu$ strain. Resolution on the order of about $1 \mu$ strain was achieved with FP approach, with up to 16 gratings multiplexed on a single fiber.

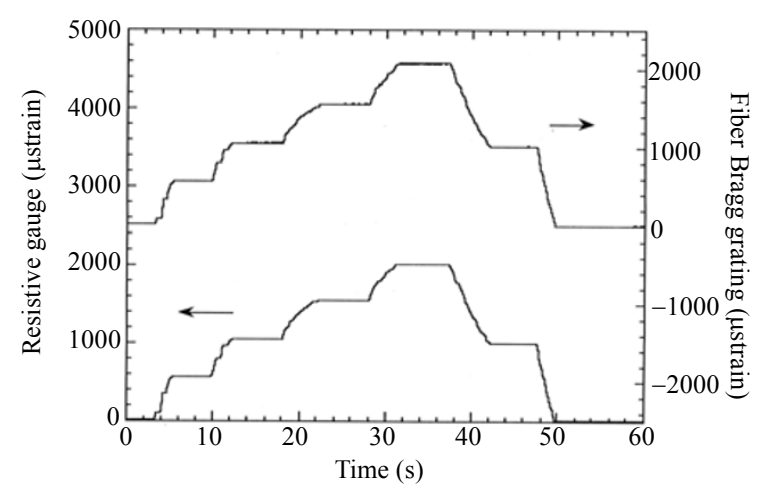

Fig. 7 Comparison of FBG strain sensor and corresponding reference electrical gauge.

A simple extension of this to address different FBG strings using an optical switch allowed such an instrumentation system to address several "arrays" of gratings. Based on this approach, a system for tracking 60 grating sensors was developed and deployed in a practical structural monitoring application [66].

While the scanning FP scans each FBG sensor wavelength in a sequential manner, direct spectroscopic analysis provides full spectrum analysis. A novel spectroscopic approach developed direct for analyzing the return signals is analysis via a fiber Fourier transform spectroscopy (FFTS) [67]. In this case, the light from an array of grating sensors is fed to an interferometer in which one arm can be scanned to change the relative optical path lengths. As the path difference passes through zero, a beat signal between the optical components is generated at the detector. For a multi-wavelength signal, such as that produced by the grating array, the detector output comprises a series of discrete audio frequencies, each of which corresponds to a particular grating.
While FBGs provide an inherent wavelengthbased multiplexing capability, approaches to augmenting this based on time and/or frequency division multiplexing were developed and demonstrated. A novel approach to implementing a high-density distributed FBG array utilized the pairing of low-reflectivity gratings with high-sensitivity time-resolved detection to form an optical time-domain reflectometer (OTDR) interrogator for FBGs was reported [68]. Because deleterious effects arise with strongly written FBG's whose reflected light signals are separated in time, but overlapping in the spectral domain (due to "multiple-reflection" and "spectral-shadowing"), this approach utilizes very low reflectivity $(<5 \%)$ gratings.

Another novel aspect of this system was the use of chirped-broadened gratings with an asymmetric "ramp" profile (sawtooth) as illustrated in Fig. 8, which produced a monotonic increase in reflectivity with strain for each of the FBGs in the sensor array. This powerful technique could be used as the basis of a very dense array of grating sensors.

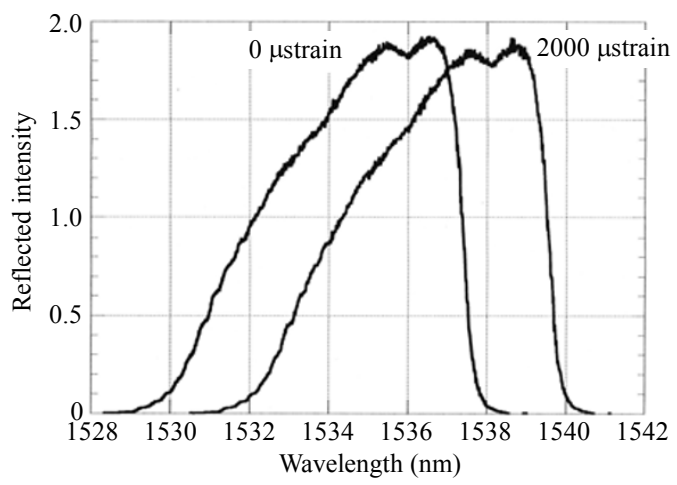

Fig. 8 Asymmetric low reflectivity "ramp" profile chirped gratings designed for OTDR-type interrogation.

The use of interferometric-configurations to detect the shift in the resonance condition of a FBG was reported in several papers $[69,70]$. Here, an unbalanced interferometer is employed as an optical filter element with an interferometric cosinusoidal transfer function, in which phase bias term depends on the input wavelength. Figure 9 shows the general 
principle of this technique: light reflected from a grating is coupled back through an all-fiber interferometer which is unbalanced by $\Delta L$. Due to the inherent wavelength dependence of the phase of an unbalanced interferometer on its input wavelength, shifts in FBG reflection wavelength are converted into phase shifts at the interferometer output.

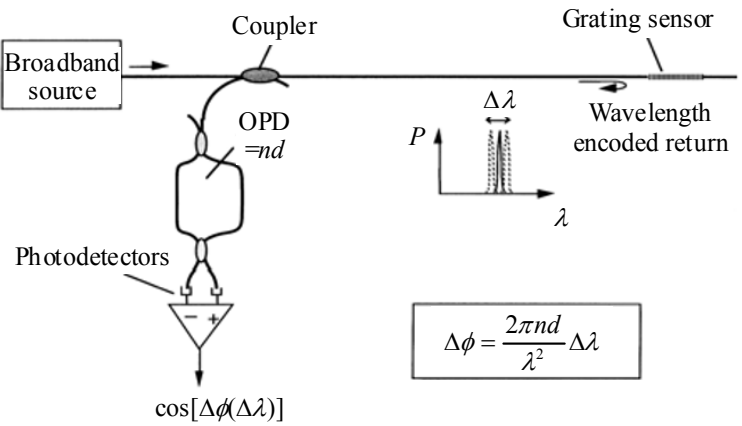

Fig. 9 Mach-Zehnder based FBG sensor interrogation for dynamic strains.

By appropriate choice of the interferometer optical path difference (OPD) (the interferometer OPD must be kept less than the effective coherence length of the light reflected from the grating approximately equal to the length of the grating), this technique can be made to be extremely sensitive to weak dynamic Bragg wavelength shifts. For example, with an interferometer of OPD of $1 \mathrm{~cm}$, the output wavelength-to-phase conversion factor is $-37 \mathrm{rad} / \mathrm{nm}$ at a wavelength of $1.3 \mathrm{~nm}$. Typical interferometric systems provide $\mu \mathrm{rad} / \mathrm{rootHz}$ phase detection sensitivity and strain resolution of -0.035 nanostrain/rootHz. A sensor with a resolution of 0.6 nanostrain/rootHz has been demonstrated. It is interesting to note that the equivalent optical frequency shift of the FBG is about $100 \mathrm{kHz}$, illustrating the ultra-high sensitivity of this approach.

Although very sensitive to dynamic strains, the interferometric technique can be problematic when being used for quasi-static strain measurement due to drifts in the interferometer bias phase itself. However, a technique to compensate for this drift using a reference wavelength to directly monitor the interferometer stability has been reported [71]. This approach has been used to monitor temperature, and in particular to allow differential temperature.

This interferometric wavelength discriminator approach was also successfully applied to a Doppler shifted laser signal reflected off a vibrating surface to provide a novel form of vibrometer [72].

Fiber Bragg gratings are also ideal for use as spectrally narrowband reflectors for creating in-fibercavities for fiber lasers. This area attracted considerable interest from both the telecommunication industry for tuneable single frequency devices for wavelength division multiplexed networks and the sensing field for strain, temperature, and very high resolution dynamic strain monitoring.

Several variations on this concept were reported [73-75]: the most basic form of a fiber Bragg grating laser sensor (FBGLS) utilizes either of two gratings of matched Bragg wavelength to create an in-fiber cavity, or one grating combined with a broadband reflector. The use of doped fiber section between the gratings or grating and reflector (e.g. Erbium) allows the system to be optically pumped to provide cavity gain and thus lasing. The device can be implemented in various ways and operated in either a single frequency or multi-mode fashion [72]. When being configured as a sensor element, changes in the environmental conditions subjected to the laser cavity and the gratings can be detected by monitoring the change in some characteristic of the output. For strain or temperature variations on the gratings themselves, the shift in wavelength of the laser output is identical to that obtained with the passive approach for monitoring FBGs. With FBG laser sensor configurations, however, it is also possible to detect such effects as the beating between different longitudinal cavity modes or polarization modes in the system [74]. As with basic FBG sensing, the inherent wavelength division addressing capabilities of gratings also allow distributed laser-sensors to be implemented [74, 75]. 
Figure 10 illustrates an example of the type of fiber laser sensors which have been demonstrated. Here, a short cavity fiber laser created by gratings of matched wavelength is remotely pumped. The grating lasers, which can be as short as $3 \mathrm{~cm}$ in length, behave as sensors with a gauge length equal to that of the cavity.

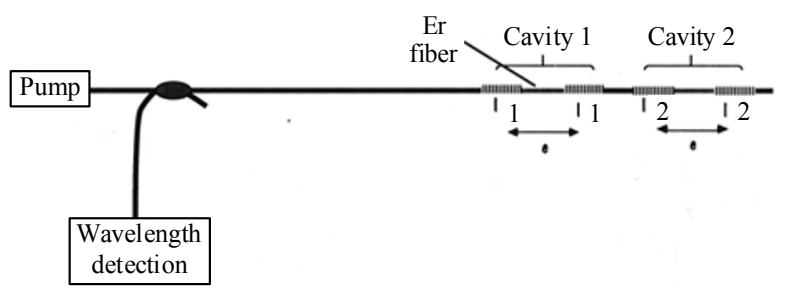

Fig. 10 Multiplexed FBG-based fiber laser sensor array.

Various configurations for FBG laser sensors have been reported, including an interferometric detection technique in which an ultra high sensitivity strain sensing was demonstrated. This configuration was used as the basis for a fiber laser magnetometer [76] and the sensitivity/crosstalk of such short gauge length strain/acoustic multiplexed sensors was investigated [77].

Other FBG grating sensors reported include long-period grating sensors [78], intra-grating sensors based on chirped FBGs with high spatial resolution capability [79], and interferometric configurations based on gratings reflectors, including novel nested interferometric topologies [80].

\subsection{Applications}

The primary focus for application of FBGs through the late 1990s and 2000s was in the development of smart structures. FBGs are ideally suited for this application, as they can be embedded into a range of materials, including composites, or surface attached in a low profile manner to other materials. A couple of notable applications included the monitoring of a US highway bridge (I-10 in New Mexico) with 60 FBGs and a remote monitoring system [81]. The gratings were bonded to steel girders on the underside of the bridge. Figure 11 shows representative sensor transient strain responses due to a passing car (Fig. 11(a)) and truck (Fig. 11(b)) loading of the bridge span (photo inset).

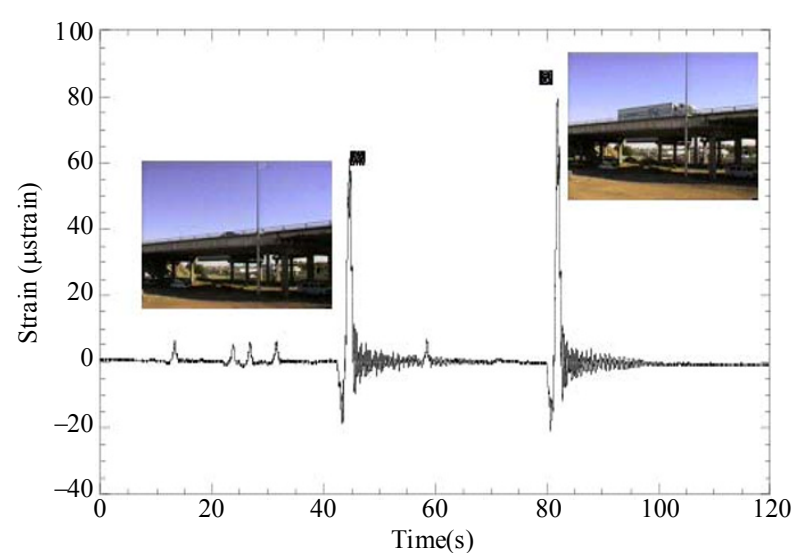

Fig. 11 FBG sensor transient strain responses due to a passing car (a) and truck (b) loading of the instrumented I-10 bridge span.

A second example of the application of distributed FBGs was on a composite ship hull. The technical program was named CHESS (composite hull embedded sensor system). In this research program, the composite hull of an advanced Norwegian Navy fast patrol boat was instrumented to monitor \& predict potential delamination damage to the hull caused by high sea loads. Particular interest on this vessel, which was a surface effect ship with a catamaran hull built of sandwich panels with a foam core and forced composite skins, was the "wet deck" between the primary dual hulls. This wet deck normally rides above the sea level, but in high sea states, the wet deck can suffer large transient loads due to wave slamming, potentially leading to hidden composite damage.

The CHESS demonstration comprised a multiplexed system monitoring 4 FBG strain sensors. The system was designed to analyze transient strain responses, as the peak loads were those that could create composite damage [82, 83]. Figure 12 shows an example of the root mean square (RMS) transient strain recorded by a grating attached to the wet deck of the ship due to a low-level wave loading events. While this initial work was conducted on a prototype vessel, this program was successfully 
transitioned to the testing of in-service ships, and more extensive FBG sensor systems, with over 50 grating sensors being installed and sea-tested [84].

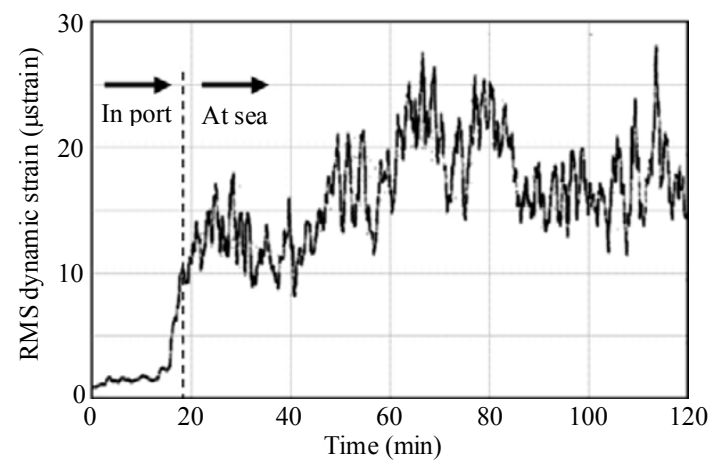

Fig. 12 FBG sensor RMS transient strain responses due to hull loading in the CHESS sea trail.

\subsection{Commercialization}

While FBGs appeared ideal for many sensing applications, little in the way of commercial sensor systems had appeared on the market through the late 1990s.

In 1999, I left NRL \& joined a new start-up, CiDRA Corporation, where we developed a suite of transducers that utilized gratings as the core building blocks for a network of wavelength-encoded sensors for pressure, temperature, vibration, and flow [85]. These devices were designed, engineered and tested to withstand the harsh environments experienced in permanently installed oil well reservoir monitoring systems. CiDRA was founded to exploit the use of FBGs in this market. The oil and gas industry was chosen as it has the right attributes to enable the fast adoption of new technology, namely, it comprised:

1)a multi \$-billion industry with very big companies,

2)an unmet need, with strong economic drivers, and

3)a track history of a strong commitment to investing in future technologies.

Down-hole, or in-well measurement plays a critical role in the production of oil and gas reservoirs: parameters such as pressure, temperature and flow provide valuable insights into the depletion dynamics of a reservoir that, if it is optimized, can be highly beneficial to the productivity and economic return. Unfortunately, oil and gas reservoirs represent some of the harshest, least accessible environments on earth. This paper addresses the development and commercialization of fiber optic sensing systems to monitor the conditions of these reservoirs on a real time basis as the oil and gas is produced.

Significant interest was directed towards this area during the late 1990s and early 2000s, and several types of fiber optic sensors were demonstrated for well monitoring use, most notably, distributed temperature sensing (DTS) for well temperature profiling. The multiplexed or distributed sensing capability of FBG sensors is of particular pertinence for in-well applications in the oil and gas industry, where there is the need to monitor a parameter, or parameters, at many spatial locations through the well-bore, or horizontal/multi-lateral components of the well are of interest.

The sensor suite developed by CiDRA comprised all facets of the "system" required for deployment in the oil \& gas industry, including armoured fiber cabling, well-head feed-throughs, sensor housings, and surface instrumentation/data logging sub-systems. The overall system is depicted in Fig. 13.

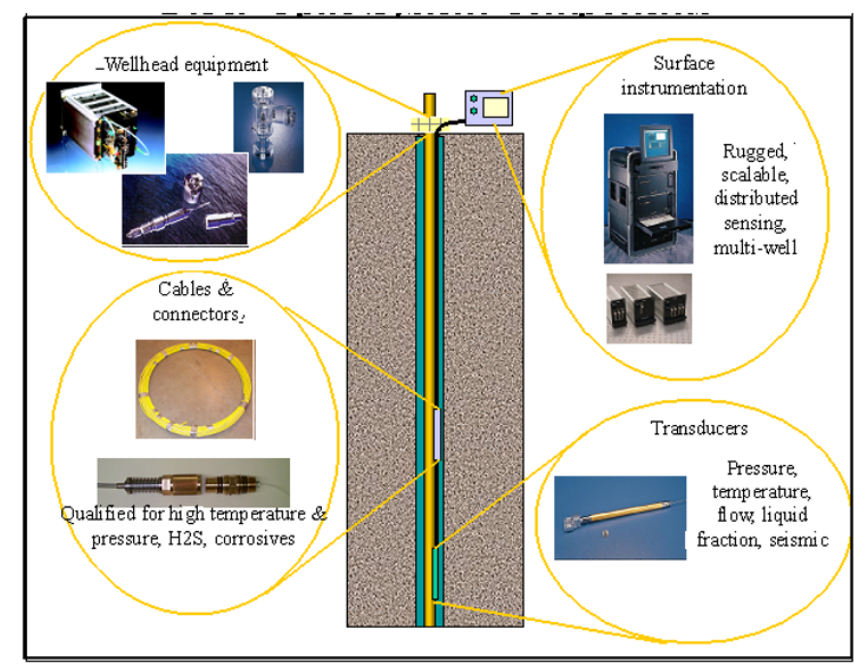

Fig. 13 CiDRA FBG-based down-hole reservoir temperature and pressure monitoring system. 
Major research and engineering activities were applied to the hardening of sensors for the temperature and pressure environments experienced in-well, which typically ranged up to $200{ }^{\circ} \mathrm{C}$ and 20,000 psi respectively. Figure 14 indicates the temperature independent nature of the output of the pressure transducer developed. Here, the sensor was cycled over a pressure range from 0 to $15,000 \mathrm{psi}$ (100 bar), with each cycle at a different temperature ranging from $25{ }^{\circ} \mathrm{C}$ to $150{ }^{\circ} \mathrm{C}$. The device showed minimal temperature sensitivity and exceptionally low hysterisis.

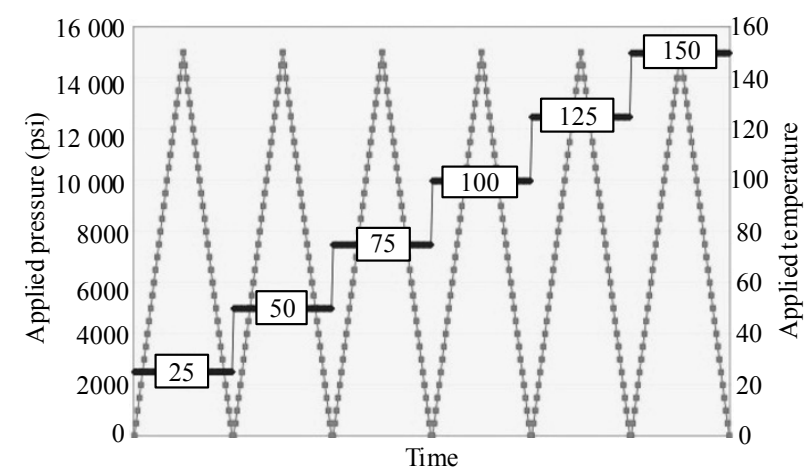

Fig. 14 FBG pressure response over 0 to 15,000 psi at various operational temperatures.

The results of efforts to minimize long term drift on a FBG pressure sensor are shown in Fig. 15. Here, the gauge was subjected to 5,000 psi applied pressure at $150{ }^{\circ} \mathrm{C}$ for over 2 years. As shown the drift in the gauge was well within $0.015 \%$ full scale/year, meeting the requirements for certain key oil-well applications. It is worthy of noting that the detection system resolution was about $0.1 \mathrm{pm}$, which corresponded to roughly $0.25 \mathrm{psi}$ for an $15,000 \mathrm{psi}$ gauge over the life of the system.

In addition to new temperature \& pressure transducers for these environments, CiDRA developed a fundamentally new methodology for multiphase flow metering. This resulted in a production-tubing deployed fiber optic flow meter which was completely non-intrusive and contained no moving parts. The flow meter utilized multiple unsteady pressure measurement spatially distributed along a sensing region of the well bore to listen to production-generated noise. Unsteady pressure measurement was monitored using an array of acoustic sensors formed via FBGs. The flow meter leveraged the common fiber optic in-well cabling etc., as the pressure \& temperature monitoring system, but utilized additional surface instrumentation.

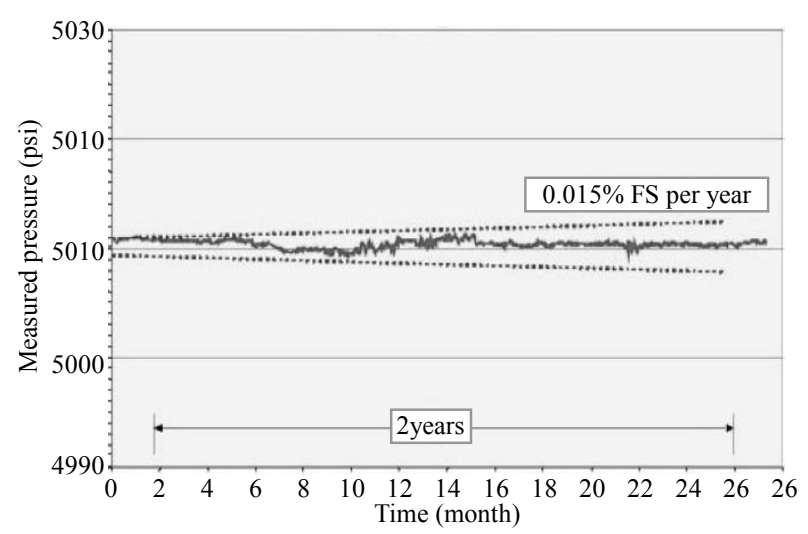

Fig. 15 FBG pressure transducer drift over 2 years at 500 psi $\& 150{ }^{\circ} \mathrm{C}$.

\section{Micro encoded-particle}

The work on Bragg gratings would not be complete without a mention of a detour into the world of bio-assays. In 2003, I moved from CiDRA to CyVera Corporation, where we developed a methodology for over-writing multiple gratings into a length of optical fiber to form a comb-like spectral signature. It was realized that if certain elements of this comb were not written, the subsequent output appeared in the form of a digital binary code in the spectral domain. While this had utility in certain sensing applications [86], we realized that the fiber should be segmented into short lengths, the "code" remained detectable in each fiber segment, and, as a photo-inscribed grating was a form of volumetric grating, these short elements could be interrogated by side illumination.

This technology ultimately led to the commercialization of a product platform for DNA analysis by Illumina Inc. in 2007 [see www.illumina.com]. 


\section{Acknowledgement}

The work reported here could not have been accomplished without the collaboration with many colleagues over the years. These are too numerous to mention by name. I am indebted to Prof. David Jackson for the opening in this field during my period at Kent afforded me.

Open Access This article is distributed under the terms of the Creative Commons Attribution License which permits any use, distribution, and reproduction in any medium, provided the original author(s) and source are credited.

\section{References}

[1] D. A. Jackson, et al., "Elimination of drift in a single-mode optical fiber interferometer using a piezoelectrically stretched coiled fiber," Appl. Opt., vol. 19, no. 17, pp. 2926-2929, 1980.

[2] D. A. Jackson, "A prototype digital phase tracker for the fiber interferometer," J. Phys. E., vol. 14, no. 11, pp. 1274-1278, 1981.

[3] A. D. Kersey, et al., "Pseudeheterodyne detection scheme for optical interferometers," Electron. Letts., vol. 18, no. 25, pp. 1081-1083, 1982.

[4] A. D. Kersey, et al., "A passive compensation scheme suitable for use in the single mode fiber interferometer," Electron. Letts., vol. 18, no. 9, pp. 392-393, 1982.

[5] A. C. Lewin, et al., "Non-contact surface vibration analysis using a monomode fiber optic interferometer incorporating an open air path," J. Phys. E., vol. 18, no. 7, pp. 604-608, 1985.

[6] A. D. Kersey, et al., "Pseudeheterodyne-detection technique for the fiber gyroscope," Electron. Letts., vol. 20, no. 9, pp. 368-370, 1984.

[7] M. Corke, et al., “All-fiber 'Michelson' thermometer," Electron. Letts., vol. 19, no. 13, pp. 471-473, 1983.

[8] A. D. Kersey, et al., "High-sensitivity fiber-optic accelerometer," Electron. Letts., vol. 18, no.13, pp. 559-561, 1982.

[9] A. D. Kersey, et al., "Detection of DC and low-frequency AC magnetic fields using an all single-mode fiber magnetometer," Electron. Letts., vol. 19, no.13, pp. 469-471, 1983.

[10] A. D. Kersey, et al., "Single mode fiber optic magnetometer with DC bias field stabilization," $J$. Lightwave Technol., vol. 3, no. 4, pp. 836-840, 1985.

[11] A. D. Kersey, D. A. Jackson, and M. Corke, "A simple fiber Fabry-Perot sensor," Optics and Lasers in Engineering, vol. 5, no. 3, pp. 141-154, 1984.

[12] M. Corke, A. D. Kersey, and D. A. Jackson, "Remote temperature sensing using polarization-preserving fiber," Electron. Lett., vol. 20, no. 2, pp. 67-69, 1984.

[13] J. D. C. Jones, D. A. Jackson, M. Corke, and A. D. Kersey, "Single mode fiber optic sensors: conventional interferometric, polarimetric and combined devices," in Proceedings, Fiber Optics'85, vol. 552, pp. 196-203, 1985.

[14] M. Corke, J. D. C. Jones, A. D. Kersey, and D. A. Jackson, "Combined Michelson and polarimetric fiber-optic interferometric sensors," Electron. Letts., vol. 21, no. 4, pp. 148-149, 1985.

[15] T. G. Giallorenzi, et al., "Optical fiber sensor technology," IEEE J. Quant. Electron., vol. 18, no. 4, pp. 626-665, 1982.

[16] A. D. Kersey, et al., "Phase compensation scheme suitable for use in single mode fiber interferometer," Electron. Lett., vol. 18, pp. 392-393, 1982.

[17] A. D. Kersey and A. Dandridge, "Phase noise reduction in coherence multiplexed interferomeric fiber sensors," Electron. Lett., vol. 22, no. 11, pp. 616-618, 1986.

[18] T. A. Berkoff, et al., "Novel analog phase tracker for interferometric fiber optic sensor applications," in SPIE Proc. on Fiber Optic and Laser Sensors VIII, vol. 1367, pp. 53-58, 1990.

[19] A. D. Kersey and R. P. Moeller, "Open loop fiber optic gryoscope with phase shift nulling signal processing," Electron. Letts., vol. 26, no. 16, pp. 1251-1253, 1990.

[20] K. J. Williams, et al., "Interferometric measurement of low frequency phase noise characteristics of a diode laser-pumped Nd:YAG ring laser," Electron. Letts., vol. 25, no.12, pp. 774-776, 1989.

[21] K. Wanser, et al., "Intrinsic thermal phase noise limit in optical fiber interferometers," Optics and Photonics News, vol. 4, no. 12, pp. 37-38, 1993.

[22] M. J. Marrone, et al., "Elimination of coherent Rayleigh backscatter induced noise in fiber Michelson interferometers," Electron. Letts., vol. 28, no. 19, pp. 1803-1804, 1992.

[23] S. K. Sheem and T. G. Giallorenzi, "Polarization effects on single mode optical fiber sensors," Appl. Phys. Lett., vol. 35, no. 12, pp. 914-917, 1979. 
[24] H. C. Lefevre, "Single-mode fiber fractional wave devices and polarization controllers," Electron. Letts., vol. 16, no. 20, pp. 778-780, 1980.

[25] A. D. Kersey, et al., "Dependence of visibility on input polarization in interferometric fiber-optic sensors," Opt. Letts., vol. 13, no. 4, pp. 288, 1988.

[26] A. D. Kersey, et al., "Optimization and stabilization of visibility in interferomeric fiber optic sensors using input polarization control," J. Lightwave Technol., vol. 6, no. 10, pp. 1599-1609, 1988.

[27] A. D. Kersey and M. J. Marrone, "Input-polarization scanning techniques for overcoming polarization-induced signal fading in interferometric fiber sensors," Electron. Letts., vol. 24, no.15, pp. 931-933, 1988.

[28] A. D. Kersey, M. J. Marrone, and A. Dandridge, "Input polarization effects on interferometric fiber-optic sensors," in Proc. SPIE, vol. 949, pp. 170-176, 1988.

[29] A. D. Kersey, et al., "Statistical modelling of polarisation induced fading in interferometric fiber sensors," Electron. Letts., vol. 27, no. 6, pp. 481-483, 1991.

[30] A. D. Kersey, et al., "Polarization-insensitive fiber optic Michelson configuration," Electron. Letts., vol. 26, pp. 518-519, 1991.

[31] M. J. Marrone and A. D. Kersey, "Visibility limits in fiber optic Michelson interferometer with birefringence compensation," Electron. Letts., vol. 27, no. 16, pp. 1422-1424, 1991.

[32] A. D. Kersey and A. Dandridge, "Monomode fiber polarization scrambler," Electron. Letts., vol. 23, no. 12, pp. 634-636, 1987.

[33] W. K. Burns and A. D. Kersey, "Fiber-optic gyroscopes with depolarized light," J. Lightwave Technol., vol. 10, no. 7, pp. 992-999, 1992.

[34] A. D. Kersey, et al., "Observation of input polarization induced phase noise in interferometric fiber optic sensors," Opt. Letts., vol. 13, no. 10, pp. 847-849, 1988.

[35] A. D. Kersey, et al., "Analysis of inputpolarization-induced phase noise in interferometric fiber-optic sensors and its reduction using polarization scrambling," J. Lightwave Technol., vol. 8, no. 6, pp. 838-845, 1990 .

[36] M. A. Davis, et al., "Reflective fiber ring resonator with polarization-independent operation," Opt. Letts., vol. 18, no. 9, pp. 750-752, 1993.

[37] A. D. Kersey, et al., "Time-division multiplexing of interferometric fiber sensors using passive phase-generated carrier interrogation," Opt. Letts., vol. 12, no. 10, pp. 775-777, 1987.
[38] A. D. Kersey, et al., "Multiplexed Mach-Zehnder ladder array with ten elements," Electron. Letts., vol. 25, no. 19, pp. 1298-1299, 1989.

[39] A. Dandridge, et al., "Multiplexing of interferometric sensors using phase generated carrier techniques," J. Lightwave Technol., vol. 5, no. 7, pp. 947-952, 1987.

[40] A. D. Kersey and A. Dandridge, "Low crosstalk code division multiplexed interferometric array," Electron. Letts., vol. 28, no. 4, pp. 351-352, 1992.

[41] A. D. Kersey and A. Dandridge, "Demonstration of a hybrid time/wavelength division multiplexed interferometric fiber sensor array," Electron. Letts., vol. 27, no. 7, pp. 554-555, 1991.

[42] A. D. Kersey and A. Dandridge, "Fiber optic multisensor networks," in Proc. Fiber Optic and Laser Sensors VI, Boston, Sept. 6-7, pp. 90-104, 1988.

[43] A. D. Kersey and A. Dandridge, "Comparative analysis of multiplexing techniques for interferometric fiber sensors," in SPIE Proc. on Fiber Optics, vol. 1120, pp. 236-246, 1989.

[44] A. D. Kersey, K. L. Dorsey, and A. Dandridge, "Demonstration of an eight-element time-division multiplexed interferometric fiber sensor array," Electron. Letts., vol. 24, no. 11, pp. 689-691, 1988.

[45] A. D. Kersey, A. Dandridge, and K. L. Dorsey, "Transmissive serial interferometric fiber sensor array," J. Lightwave Technol., vol. 7, no. 5, pp. 846-854, 1989.

[46] A. D. Kersey, et al., "Cross talk in a fiber-optic Fabry-Perot sensor array with ring reflectors," Opt. Letts., vol. 14, no. 1, pp. 93-95, 1989.

[47] A. D. Kersey and A. Dandridge, "Dual wavelength approach to interferometric sensing," in SPIE Proc. on Soc. Photo-Opt. Instrum. Eng., vol. 798, pp. 176-181, 1987.

[48] A. D. Kersey, et al., "Two-wavelength fiber gyroscope with wide dynamic range," Electron. Lett., vol. 22, no. 18, pp.935-937, 1986.

[49] A. D. Kersey, et al., "Differential polarmetric fiber-optic sensor configuration with dual wavelength operation," Appl. Opt., vol. 28, no. 2, pp. 204-206, 1989.

[50] C. A. Wade, et al., "Temperature sensor based on a fiber-optic differential delay RF filter," Electron. Letts., vol. 24, no. 21, pp. 1305-1306, 1988.

[51] T. A. Berkoff, et al., "Interferometric fiber displacement/strain sensor based on source coherence synthesis," Electron. Letts., vol. 26, no. 7, pp. 452-453, 1990.

[52] A. D. Kersey, F. Bucholtz, and A. Dandridge, "New nonlinear phase transduction method for $\mathrm{dc}$ 
measurand interferometric fiber sensors," Electron, Letts., vol. 22, no. 2, pp. 75-76, 1986.

[53] F. Bucholtz, A. D. Kersey, and A. Dandridge, "DC fiber-optic accelerometer with sub-pg sensitivity," Electron. Letts., vol. 22, no. 9, pp. 451-453, 1986.

[54] A. D. Kersey, F. Bucholtz, K. Sinansky, and A. Dandridge, "Interferometric sensors for dc measurands - a new class of fiber sensors," in SPIE Proc. on Fiber Optic and Laser Sensors IV, vol. 718, pp. 198-202, 1986.

[55] C. A. Wade, et al., "Multiplexing of sensors based on fiber-optic differential delay RF filters," Electron. Letts., vol. 24, no. 25, pp. 1557-1559, 1988.

[56] F. Bucholtz, A. D. Kersey, and A. Dandridge, "Multiplexed nonlinear interferometric fiber sensors," in OFS'86 Tech. Dig., pp. 63-64, 1986.

[57] A. D. Kersey and A. Dandridge, "Optical fiber Faraday rotation current sensor with closed-Loop operation," Electron. Lett., vol. 21, no. 11, pp. 464-466, 1985.

[58] A. D. Kersey and D. A. Jackson, "Current sensing utilizing heterodyne detection of the Faraday effect in single-mode optical fiber," J. Lightwave Technol., vol. 4, no. 6, pp. 640-644, 1986.

[59] A. D. Kersey, et al., "Sensitivity-bandwidth limitations in optical-fiber Faraday-rotation current sensors," International Journal of Optoelectronics, vol. 3, pp. 323-332, 1988.

[60] S. T. Vorha, et al., "Fiber-optic DC and low frequency electric field sensor," Opt. Letts., vol. 16, no. 18, pp. 1445-1447, 1991.

[61] M. J. Marrone, et al., "Fiber-optic magnetic field sensor with an orthoconjugating loop mirror," Opt. Letts., vol. 18, no. 18, pp. 1556-1558, 1993.

[62] A. D. Kersey, et al., "Fiber grating sensors," $J$. Lightwave Technol., vol. 15, no. 8, pp. 1442-1463, 1997.

[63] G. Meltz, W. W. Morey, and W. H. Glenn, "Formation of Bragg gratings in optical fibers by a transverse holographic method," Opt. Letts., vol. 14, no. 15 , pp. 823-825, 1989.

[64] S. M. Melle, K. Liu, and R. M. Measures, “A passive wavelength demodulation system for guided-wave Bragg grating sensors," IEEE Photonics Technol. Letts., vol. 4, no. 5, pp. 516-518, 1992.

[65] A. D. Kersey, et al., "Multiplexed fiber Bragg grating strain sensor system with a fiber Fabry Perot wavelength filter," Opt. Letts., vol. 18, no. 16, pp. 1370-1372, 1993.

[66] M. A. Davis, et al., "Interrogation of 60 fiber Bragg grating sensors with $\mu$ strain resolution capability," Electron. Letts., vol. 32, no. 15, pp. 1393-1394, 1996.
[67] M. A. Davis and A. D. Kersey, "Application of a fiber Fourier transform spectrometer to the detection of wavelength-encoded signals from Bragg grating sensors," J. Lightwave Technol., vo. 13, no. 7, pp. 1289-1295, 1995.

[68] A. D. Kersey, M. A. Davis, and T. Tsai, "Fiber optic Bragg grating strain sensor with direct reflectometric interrogation," in Proc. 11th. Int. Conf. on Optical Fiber Sensors, OFS'96, Sapporo, Japan, May 21, pp. 634-636, 1996.

[69] A. D. Kersey, T. A. Berkoff, and W. W. Morey, "High resolution fiber Bragg grating based strain sensor with interferometric wavelength shift detection," Electron. Letts., vol. 28, no. 3, pp. 236-238, 1992.

[70] A. D. Kersey, T. A. Berkoff, and W. W. Morey, "Fiber optic Bragg grating strain sensor with drift-compensated high resolution interferometric wavelength shift detection," Opt. Letts., vol. 18, no. 1, pp. 72-74, 1993.

[71] A. D. Kersey and T. A. Berkoff, "Fiber optic Bragg grating differential temperature sensor," Photon. Technol. Letts., vol. 4, no. 10, pp. 1183-1185, 1992.

[72] L. Fabiny and A. D. Kersey, "Interferometric fiber-optic Doppler velocimeter with high-dynamic range," Photon. Technol. Letts., vol. 9, no. 1, pp. 79-81, 1997.

[73] A. D. Kersey and W. W. Morey, "Multi-element Bragg grating based fiber laser strain sensor," Electron. Letts., vol. 29, no. 11, pp. 964-966, 1993.

[74] A. D. Kersey and W. W. Morey, "Multiplexed Bragg grating fiber laser strain sensor system with mode-locked interrogation," Electron. Letts., vol. 29, no. 1, pp. 112-114, 1993.

[75] K. P. Koo and A. D. Kersey, "Bragg grating based laser sensor systems with interferometric interrogation and wavelength division multiplexing," J. Lightwave Technol., vol. 13, no. 7, pp. 1243-1249, 1995.

[76] K. P. Koo, et al., "Bragg grating based laser magnetometer," presented at Postdeadline Presentation, CLEO'95, 1995.

[77] K. P. Koo and A. D. Kersey, "Noise and crosstalk of a 4-element serial fiber laser sensor array," in Tech. Dig. Conf. Opt. Fiber Commun., San Jose, CA, Feb. 25, vol. 2, pp. 226, 1996.

[78] H. J. Patrick, G. M. Williams, A. D. Kersey, J. R. Pedrazzani, and A. M. Vengsarkar, "Hybrid fiber Bragg grating/long period fiber grating sensor for strain/temperature discrimination," Photon. Technol. Letts., vol. 8, no. 9, pp. 1223-1225, 1996.

[79] M. J. Marrone, A. D. Kersey, and M. A. Davis, "Fiber sensors based on chirped Bragg gratings," 
presented at OSA Annual Meeting, Paper WGG5, Rochester, New York, Oct., 1996.

[80] A. D. Kersey and M. J. Marrone, "Nested interferometric sensors utilizing fiber Bragg grating reflectors," in Proc. 11th. Int. Conf. on Optical Fiber Sensors, OFS'96, Sapporo, Japan, May, pp. 618-621, 1996.

[81] A. D. Kersey, M. A. Davis, and D. G. Bellemore, "Development of fiber sensors for structural monitoring," in Proc. SPIE on Nondestructive Evaluation of Aging Bridges and Highways, vol. 2456, pp. 262-268, 1995.

[82] A. D. Kersey, M. A. Davis, T. A. Berkoff, A. Dandridge, R. T. Jones, T. Tsai, G. Cogdell, G. Wang, G. B. Havsgârd, K. Pran, and S. Knudsen, "Transient load monitoring on a composite hull ship using distributed fiber optic bragg grating sensors," in Proc. SPIE on Smart Structures and Materials, vol. 3042, pp. 421-430, 1997.
[83] G. Wang, G. Havsgárd, E. Urnes, K. Pran, S. Knudsen, A. D. Kersey, M. A. Davis, T. A. Berkoff, A. Dandridge, R. T. Jones, T. Tsai, and G. Cogdell, "Digital demodulation and signal processing applied to fiber Bragg grating strain sensor arrays in monitoring transient loading effects on ship hulls," in 12th mt Conf. on Optical Fiber Sensors, pp. 612-615, 1997.

[84] G. A. Johnson, K. Pran, G. Wang, G. B. Havsgârd, and S. Vohra, "Structural monitoring of a composite hull air cushion catamaran with a multi-channel fiber bragg grating sensor system," in Structural health monitoring 2000, pp. 190-198, 1999.

[85] A. D. Kersey, "Optical fiber sensors for downwell monitoring applications in the oil and gas industry," in Proc. OFS'13, pp. 326-331, 1999.

[86] A. D. Kersey, et al., "Bragg grating sensor system with spectral response or code division multiplexing," United States Patent, Patent \# 6,489,606, Dec. 3, 2002. 